\title{
COMPARACIÓN DE ESTRATEGIAS DE CONTROL PARA SISTEMAS MPPT: OPTIMIZACIÓN DE LA POTENCIA ENTREGADA A LA CARGA VS OPTIMIZACIÓN DE LA POTENCIA EXTRAÍDA DEL GENERADOR FOTOVOLTAICO
}

\author{
Ríos J. \\ Departamento de Ingeniería Electrónica, de Sistemas Informáticos y Automática. Universidad de Huelva \\ (Spain),rios@uhu.es \\ Enrique J. M. \\ Departamento de Ingeniería Electrónica, de Sistemas Informáticos y Automática. Universidad de Huelva \\ (Spain), juanm.enrique@diesia.uhu.es \\ Barragán A. J. \\ Departamento de Ingeniería Electrónica, de Sistemas Informáticos y Automática. Universidad de Huelva \\ (Spain), antonio.barragan@diesia.uhu.es \\ Andújar J. M. \\ Departamento de Ingeniería Electrónica, de Sistemas Informáticos y Automática. Universidad de Huelva \\ (Spain), andujar@uhu.es
}

\begin{abstract}
Resumen
Los sistemas de seguimiento del punto de máxima potencia (MPPT) tratan de maximizar el rendimiento de una instalación fotovoltaica mediante el ajuste dinámico del ciclo de trabajo $(\delta)$ del convertidor CC/CC empleado para conectar el generador con la carga. Tradicionalmente, el algoritmo de control empleado para la definición del ciclo de trabajo determina el valor de este parámetro con objeto de maximizar la potencia generada por el generador fotovoltaico. Estas técnicas no tienen en cuenta el carácter no ideal del convertidor $\mathrm{CC} / \mathrm{CC}$, cuyo rendimiento real depende también del ciclo de trabajo ( $\delta$ ) con el que opera. Considerando las características del convertidor CC/CC no puede afirmarse, a priori, que el método que optimiza la generación de potencia del generador fotovoltaico maximiza también la entrega de potencia en la carga, objetivo final de un sistema MPPT.
\end{abstract}

Este trabajo adapta el ampliamente extendido algoritmo "Perturbación/Observación, $P \& O$ " para optimizar la entrega de potencia en la carga, midiendo las variables que determinan el comportamiento del algoritmo a la salida del convertidor CC/CC, y compara el rendimiento obtenido con el que se obtiene con el planteamiento clásico de optimización de la potencia entregada por el generador fotovoltaico, en el que la medida de las variables que determinan el funcionamiento del algoritmo se realizan en la entrada del convertidor CC/CC.

Se ha analizado un sistema fotovoltaico que emplea un convertidor boost para conectar el generador fotovoltaico (PVG) con la carga, el cual ha sido sometido a condiciones atmosféricas variables, en cuanto a radiación y temperatura, y a distintos requerimientos de consumo de carga, obteniendo los rendimientos ofrecidos por el sistema para las dos filosofias de control. Estos resultados se discuten para determinar si hay una pérdida significativa de rendimiento en el planteamiento clásico frente a la nueva propuesta y la conveniencia de desarrollar sistemas que optimicen la potencia útil, esto es, la entregada a la carga.

Palabras clave: MPPT, Convertidor, Ciclo de trabajo, Generador fotovoltaico, Eficiencia.

\section{INTRODUCCIÓN}

Las curvas $\mathrm{I}-\mathrm{V}$ y $\mathrm{P}-\mathrm{V}$ de un generador fotovoltaico muestran un aspecto como el que se muestra en la figura 1.

Como puede observarse, hay un punto, MPP (Maximum Power Point), en el que la potencia entregada por el generador es máxima. Se sabe que 

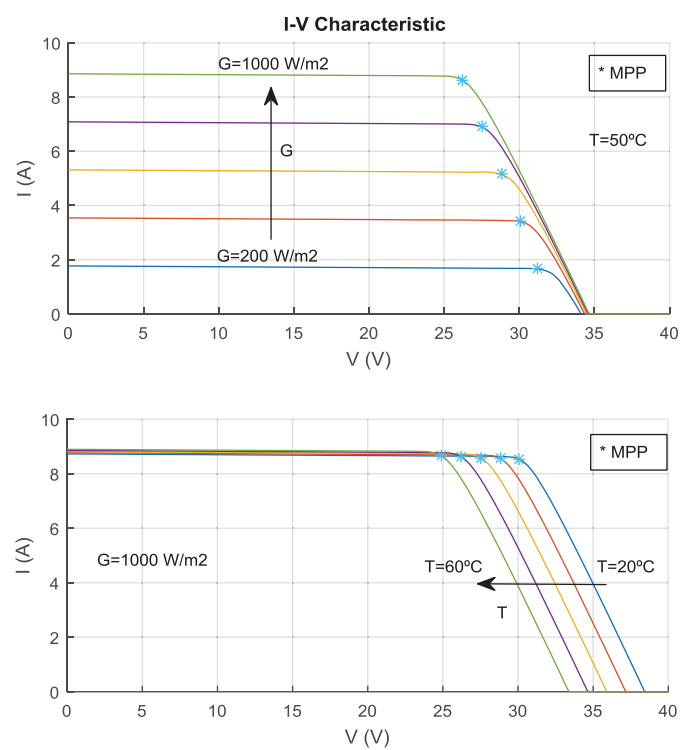
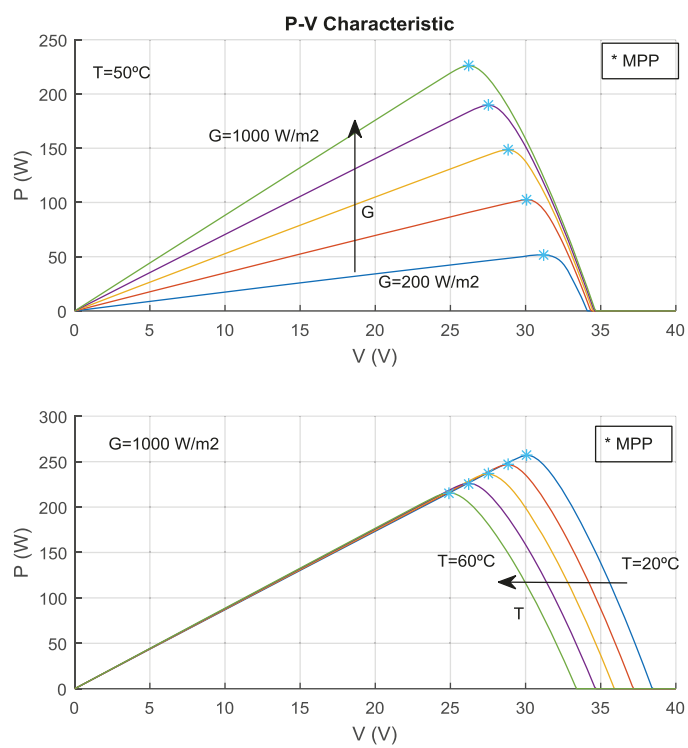

Figura 1. Característica $I / V$ y $P / V$ para diferentes valores de radiación y temperatura

estas curvas muestran dependencias significativas con la temperatura $\mathrm{T}$ y la radiación $\mathrm{G}$ que inciden en la matriz fotovoltaica, por lo que el MPP del PVG dependerá de G y T. Por lo tanto, el MPP no es un punto estático, sino que describe una trayectoria durante el día. Esta trayectoria depende de las variaciones de $\mathrm{G}$ y $\mathrm{T}$.

Para optimizar el funcionamiento de las instalaciones fotovoltaicas, se utilizan los sistemas de seguimiento del punto de máxima potencia (MPPT), que intentan situar la instalación en su punto de máxima potencia. Un sistema MPPT consiste básicamente en un convertidor de $\mathrm{CC} / \mathrm{CC}$ ubicado entre el generador fotovoltaico $\mathrm{y}$ la carga que funciona como un adaptador de impedancia. Este convertidor se rige por un algoritmo que determina el ciclo de trabajo $(\delta)$ para que el sistema funcione de manera eficiente [13].

Tradicionalmente, el criterio de posicionamiento de estos sistemas es maximizar la potencia entregada por el generador [1-10], sin embargo, este trabajo tiene como objetivo mostrar la validez del criterio de maximizar la potencia entregada a la salida del convertidor DC/DC, esto es en la carga del sistema, lo que podría llamarse potencia útil.

Los sistemas tradicionales presuponen una eficiencia (rendimiento) constante para el convertidor $\mathrm{CC} / \mathrm{CC}$, obviando su dependencia con el ciclo de trabajo. Por lo tanto, un máximo de potencia en la entrada del convertidor (salida PVG) debe corresponder a un máximo en la salida del mismo (potencia en la carga). Sin embargo, una publicación reciente [11] detalla exhaustivamente la dependencia del rendimiento de un convertidor $\mathrm{CC} / \mathrm{CC}$ con el ciclo de trabajo cuando sus pérdidas debidas a las resistencias parásitas asociadas con el cableado de la instalación, el inductor, el diodo y el transistor son consideradas. Estas curvas de rendimiento no son planas, mostrando una fuerte dependencia del ciclo de trabajo, por lo tanto, un máximo de potencia en la entrada del convertidor $\mathrm{CC} / \mathrm{CC}$ no tiene por qué resultar en una potencia máxima en la salida del mismo (si el rendimiento es bajo para ese ciclo de trabajo).

Puesto que los convertidores $\mathrm{CC} / \mathrm{CC}$ son parte fundamental en los sistemas MPPT, se hace necesario considerar este nuevo aspecto. Por tanto, o se tienen modelos muy precisos del funcionamiento del convertidor, o se cambia su consigna de funcionamiento (objeto de este trabajo).

De entre los métodos más usuales de seguimiento del MPP el archiconocido P\&O (Perturb \& Observed) $[3,4]$ es el más usado debido principalmente a su simplicidad y robustez. En este trabajo se propone estudiar el comportamiento de un sistema fotovoltaico gobernado por un $\mathrm{P} \& \mathrm{O}$ tradicional, pero en vez de maximizar la potencia a la salida del PVG, se maximiza la potencia entregada a la carga, esto es, a la salida del $\mathrm{CC} / \mathrm{CC}$. En la práctica, esta modificación es tan simple como cambiar el/los sensores de potencia a la salida del $\mathrm{CC} / \mathrm{CC}$.

Tras esta introducción, en la sección 2 se describen los datos y métodos utilizados para este trabajo, en la sección 3 se detallan las simulaciones realizadas y por último, en la sección 4 se presentan las conclusiones más importantes. 


\section{DATOS Y MÉTODOS}

Para el análisis efectuado en este trabajo, se ha considerado una instalación fotovoltaica compuesta por un generador fotovoltaico mas un convertidor DC/DC y una carga resistiva. El generador fotovoltaico es el modelo ISF 250 cuyas características se muestran en la tabla 1.

Tabla 1. Parámetros del panel fotovoltaico ISF-250

\begin{tabular}{|l|l|}
\hline Parámetro & Descripción \\
\hline $\mathrm{A}=1.2$ & Ideality factor of PN junction \\
\hline $\mathrm{Eg}=1.12 \mathrm{eV}$ & Band gap energy \\
\hline $\mathrm{n}_{\mathrm{p}}=6$ & $\mathrm{~N}^{\circ}$ de módulos conectados en paralelo \\
\hline $\mathrm{n}_{\mathrm{s}}=10$ & $\mathrm{~N}^{\mathrm{o}}$ de células conectadas en serie \\
\hline $\mathrm{P}_{\max }=250 \mathrm{~W}$ & $\begin{array}{l}\text { Potencia máxima en condiciones } \\
\text { estándar }\end{array}$ \\
\hline $\mathrm{V}_{\max }=30.6 \mathrm{~V}$ & $\begin{array}{l}\text { Tensión en el punto de máxima } \\
\text { potencia }\end{array}$ \\
\hline $\mathrm{I}_{\mathrm{max}}=8.17 \mathrm{~A}$ & $\begin{array}{l}\text { Corriente en el punto de máxima } \\
\text { potencia }\end{array}$ \\
\hline $\mathrm{NOTC}=45^{\circ} \mathrm{C}$ & $\begin{array}{l}\text { Temperatura nominal de operación de } \\
\text { célula }\end{array}$ \\
\hline $\mathrm{I}_{\mathrm{sc}}=8.75 \mathrm{~A}$ & $\begin{array}{l}\text { Corriente de cortocircuito en } \\
\text { condiciones estándar }\end{array}$ \\
\hline $\mathrm{V}_{\mathrm{oc}}=37.8 \mathrm{~V}$ & $\begin{array}{l}\text { Tensión a circuito abierto en } \\
\text { condiciones estándar }\end{array}$ \\
\hline $\begin{array}{l}\mathrm{K}_{\mathrm{v}}=-0.323 \\
\%^{\circ} /{ }^{\circ} \mathrm{C}\end{array}$ & Coeficiente de temperatura de $\mathrm{V}_{\mathrm{oc}}$ \\
\hline $\mathrm{K}_{\mathrm{i}}=0.042 \% /{ }^{\circ} \mathrm{C}$ & Coeficiente de temperatura de $\mathrm{I}_{\mathrm{sc}}$ \\
\hline $\mathrm{R}_{\mathrm{s}}=0.82 \Omega$ & Resistencia serie \\
\hline $\mathrm{R}_{\mathrm{p}}=324 \Omega$ & Resistencia paralelo \\
\hline & Condiciones estándar: $25^{\circ} \mathrm{C}$ y $1000 \mathrm{~W} / \mathrm{m}^{2}$ \\
\hline
\end{tabular}

Para las simulaciones se ha empleado el modelo de una exponencial y 5 parámetros descrito en [1].

El convertidor utilizado es del tipo Boost como el mostrado en la figura 2. Los parámetros fundamentales de este convertidor se muestran en la Tabla 2.

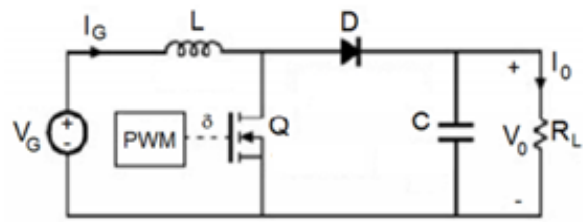

Figura 2: Convertidor boost empleado

Tabla 2. Parámetros del convertidos DC/DC

\begin{tabular}{|l|l|}
\hline Parámetro & Descripción \\
\hline $\mathrm{V}_{\Upsilon}=0.7 \mathrm{~V}$ & Tensión directa del diodo \\
\hline $\mathrm{r}=0.5 \Omega$ & Resistencia de la inductancia \\
\hline $\mathrm{R}_{\mathrm{S}}=0.3 \Omega$ & Resistencia en conducción \\
\hline $\mathrm{R}_{\mathrm{D}}=0.3 \Omega$ & Resistencia directa del diodo \\
\hline $\mathrm{L}=1 \mathrm{mHr}$ & Valor de la inductancia \\
\hline $\mathrm{f}=10 \mathrm{KHz}$ & Frecuencia de trabajo \\
\hline $\mathrm{R}_{\mathrm{off}}=1.5 \Omega$ & Resistencia parásita de la inductancia \\
\hline
\end{tabular}

La resistencia $R_{\text {off }}$ engloba a las resistencias parásitas en serie con el inductor, en las que juega un papel significativo la debida a los cables de conexión del generador fotovoltaico.

La carga empleada es siempre resistiva pero se han considerado los casos de carga constante y variable.

Los datos de radiación y temperatura empleados para este análisis corresponden a datos reales muestreados en un día de octubre del suroeste de la Península Ibérica. Estos datos se muestran en la figura 3. El día elegido corresponde a un día con un comportamiento más o menos estable con alguna perturbación significativa.

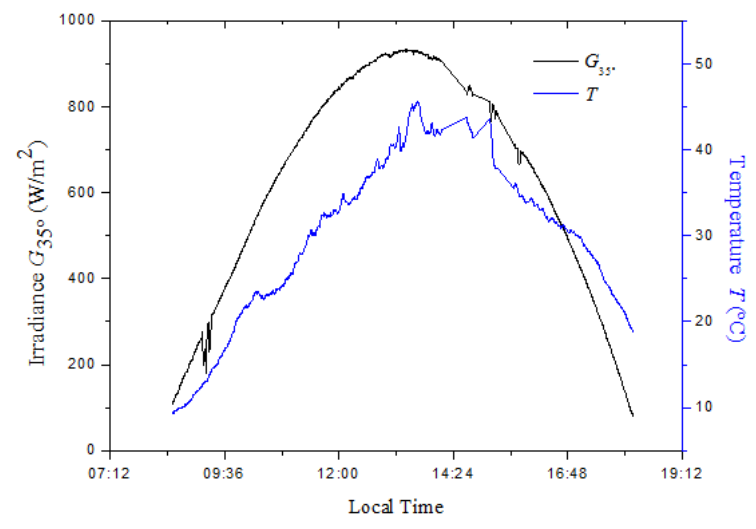

Figura 3: Datos de radiación y temperatura empleados para el análisis

Para la medida del rendimiento se ha utilizado la siguiente expresión (1) $[1,4,12]$ :

$$
\eta=\frac{\int_{0}^{t} P_{\text {inst }}(t) d t}{\int_{0}^{t} P_{r e a l-M P P}(t) d t}
$$

donde $\mathrm{P}_{\text {inst }}(\mathrm{t})$ es la potencia suministrada por el generador fotovoltaico controlado por el MPPT bajo estudio y $\mathrm{P}_{\mathrm{MPP}}(\mathrm{t})$ la potencia del MPP actual para las condiciones de radiación y temperatura dadas en el instante de tiempo evaluado.

\section{SIMULACIONES}

Se han realizado dos simulaciones con el mismo sistema fotovoltaico. La primera de las simulaciones se ha realizado considerando que la carga conectada al sistema es constante de valor $50 \Omega$. Para la segunda simulación se ha considerado que la carga 
del sistema puede variar de forma aleatoria pudiendo tomar los valores 30,50 y $70 \Omega$. En la figura 4 se muestra el perfil de variación de carga al que se ha sometido la instalación fotovoltaica para el caso de carga variable.

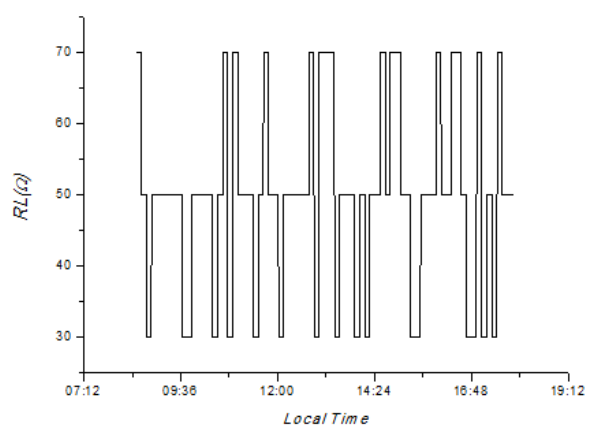

Figura 4: Perfil de variación de carga

En las figuras 5 y 6 se muestran los resultados obtenidos respectivamente para carga fija y variable. En ellas, $P \& O$ classic hace referencia al enfoque tradicional de medida de la potencia en la salida del generador, mientras que $P \& O$ modified se refiere a la medida de la potencia en la carga.

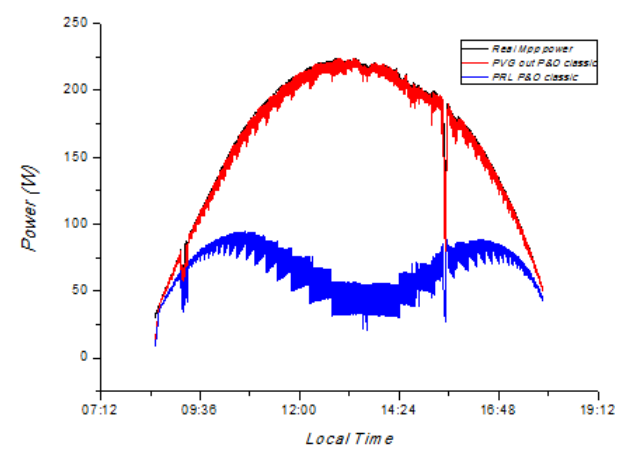

Fig. 5 a. Curvas de potencia $P \& O$ classic

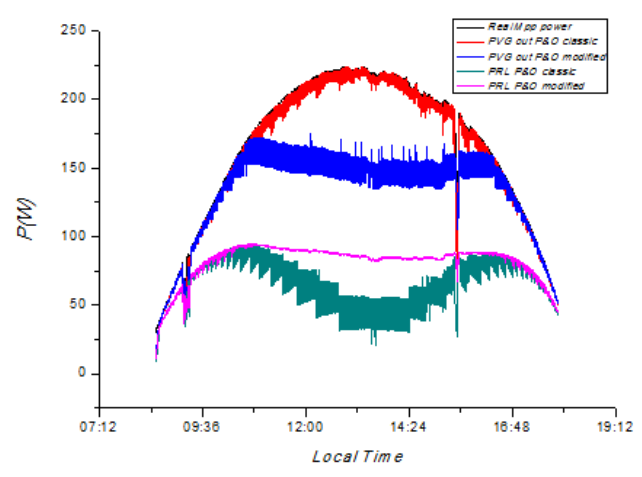

Fig. 5 c. Curvas de potencia, ambos casos
La primera gráfica de cada figura (5.a y 6.a) muestra la potencia máxima que podría extraerse del generador, la que consigue obtener el algoritmo $\mathrm{P} \& \mathrm{O}$ clásico y la entregada a la carga, mientras que la segunda (5.b y 6.b) muestra las potencias en el caso del algoritmo $\mathrm{P} \& \mathrm{O}$ modificado. En estas gráficas puede observarse la disipación de potencia en el convertidor (diferencia comprendida entre las curvas de potencia extraída del generador y entregada a la carga). Además, puede observarse como, en las horas centrales del día donde la radiación incidente es mayor, se entrega a la carga menos potencia que en otros momentos de menor incidencia. Esto es debido a los altos valores de $\delta$ que genera el algoritmo $\mathrm{P} \& \mathrm{O}$ para tratar de extraer la máxima potencia del panel en el enfoque clásico. En el planteamiento modificado este efecto se ve compensado ya que el algoritmo trata de maximizar la potencia que entrega a la carga y no la extraída del panel. En la tercera gráfica (5.c y 6.c) se representan conjuntamente las curvas de potencias para el algoritmo clásico y modificado. Puede observarse como el algoritmo P\&O modificado entrega a la carga más potencia que el clásico pese a extraer menos potencia del generador. Finalmente, la cuarta gráfica (5.d y 6.d) muestra los ciclos de servicio y rendimientos del convertidor tanto para el algoritmo P\&O clásico como para el

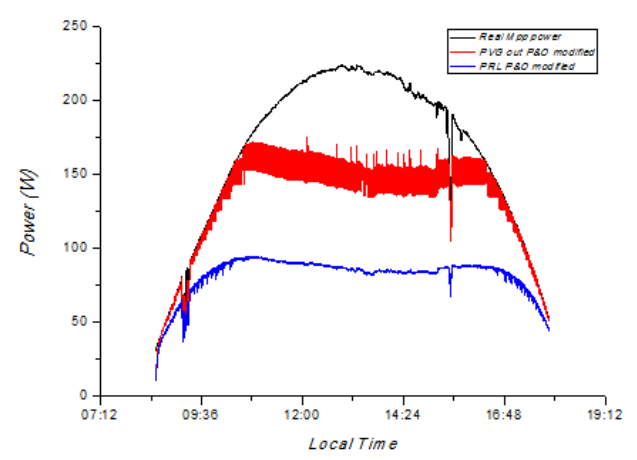

Fig. 5 b. Curvas de potencia $P \& O$ modified

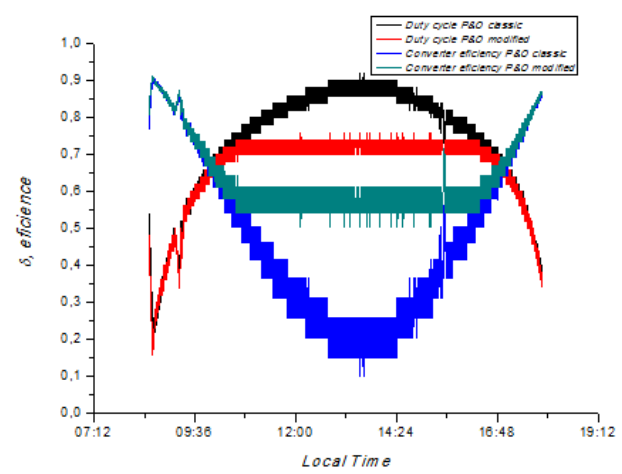

Fig. 5 d. Ciclo de trabajo y eficiencia del convertidor, ambos casos

Figura 5. Curvas de potencia, ciclo de trabajo y eficiencia del convertidor. Caso de carga fija 


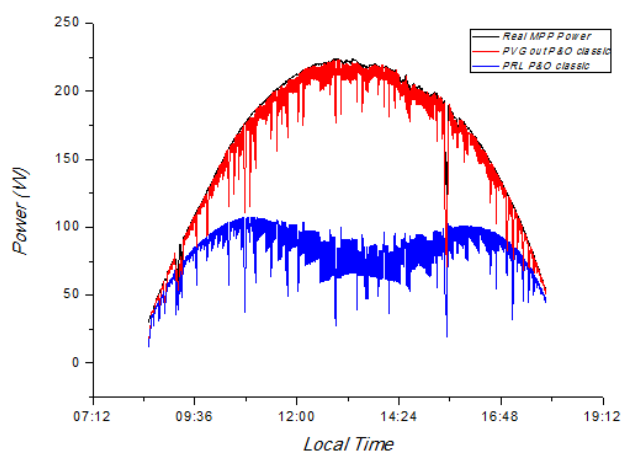

Fig. 6 a. Curvas de potencia $P \& O$ classic

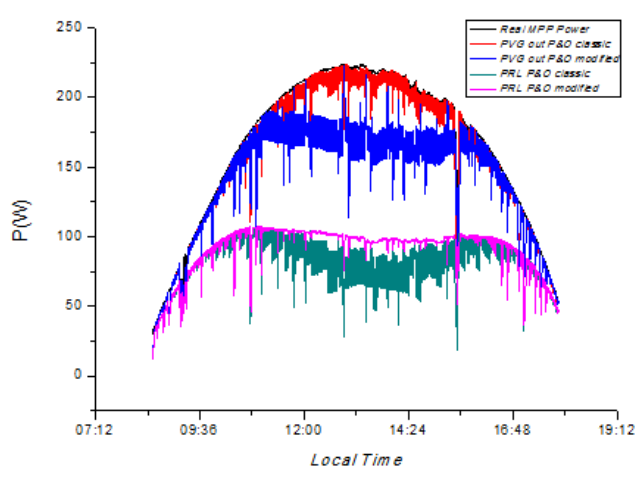

Fig. 6 c. Curvas de potencia, ambos casos

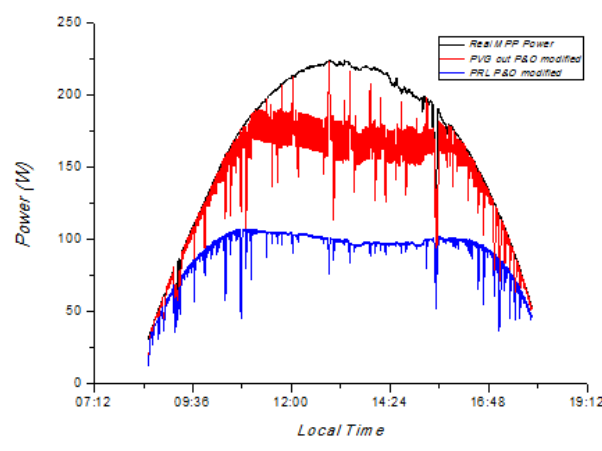

Fig. 6 b. Curvas de potencia $P \& O$ modified

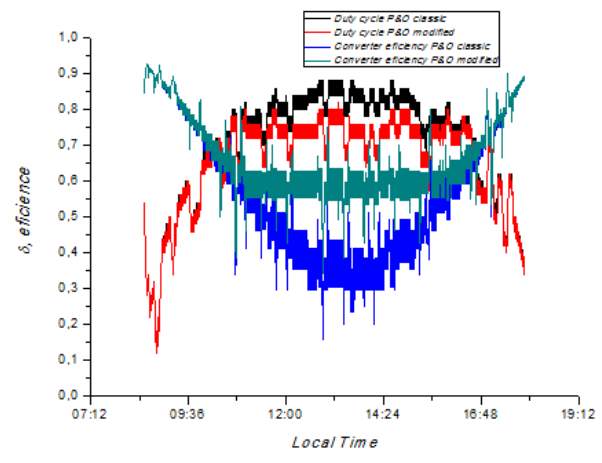

Fig.6 d. Ciclo de trabajo y eficiencia del convertidor, ambos casos

Figura 6. Curvas de potencia, ciclo de trabajo y eficiencia del convertidor. Caso de carga variable

modificado. Esta gráfica deja de manifiesto la relación entre los altos valores de $\delta$ y la reducción de eficiencia del convertidor. Se observa como en el caso del $\mathrm{P} \& \mathrm{O}$ modificado $\delta$ no supera cierto valor que corresponde a aquel en que el rendimiento del convertidor cae drásticamente. El algoritmo clásico es incapaz de detectar y tener en cuenta este valor de $\delta$.

La tabla 3 muestra los rendimientos obtenidos por la instalación fotovoltaica completa bajo los dos enfoque de medida de potencia tanto para carga fija como para carga variable. Se indica además el incremento de rendimiento obtenido al medir la potencia en la carga.

\section{CONCLUSIONES}

De los resultados de las simulaciones podemos extraer las siguientes conclusiones:

- El convertidor juega un papel muy importante en el sistema fotovoltaico. Es conveniente considerar sus características ya que determinan en gran medida el resultado obtenido. Si se tienen en cuenta sus características se observa que el rendimiento obtenido por el sistema fotovoltaico es sensiblemente inferior al que se obtendría si no se considerase. Típicamente, el rendimiento que se obtiene al simular un sistema fotovoltaico gobernado por el algoritmo P\&O supera el $90 \%$ cuando no se consideran las características del convertidor.

- En el cómputo global, medir la potencia en la carga

Tabla 3. Rendimientos obtenidos por el algoritmo $\mathrm{P} \& \mathrm{O}$

\begin{tabular}{|c|c|c|c|c|c|}
\hline \multicolumn{4}{|c|}{ Caso de carga constante. $\mathrm{RL}=50 \Omega$} & \multicolumn{2}{c|}{ Caso de carga variable (30,50,70 $\Omega)$} \\
\hline $\begin{array}{c}\text { Perform. measuring the } \\
\text { power at output of the PVG } \\
(\%)\end{array}$ & $\begin{array}{c}\text { Perform. measuring the } \\
\text { power at the load (\%) }\end{array}$ & $\begin{array}{c}\text { Perform. gain when } \\
\text { measuring power at the } \\
\text { load (\%) }\end{array}$ & $\begin{array}{c}\text { Perform. measuring the } \\
\text { power at output of the } \\
\text { PVG (\%) }\end{array}$ & $\begin{array}{c}\text { Perform. measuring the } \\
\text { power at the load (\%) }\end{array}$ & $\begin{array}{c}\text { Perform. gain when } \\
\text { measuring power at the load } \\
(\%)\end{array}$ \\
\hline 49.82 & 55.34 & 5.52 & 48.82 & 54.12 \\
\hline
\end{tabular}


en vez de hacerlo en la salida del generador permite conseguir una mejora significativa en el rendimiento del sistema. En cualquier caso, la potencia entregada en la carga midiendo en la misma es siempre igual o superior a la entregada si medimos en la salida del generador.

- La resistencia de carga parece no influir en el rendimiento obtenido en ninguno de los dos casos. Tanto trabajando con una carga fija como con una variable los resultados son similares. Este aspecto merece ser estudiado con más detalle.

- Finalmente, se observa que al medir la potencia en la carga, la potencia entregada a la misma presenta un rizado sensiblemente inferior al que tiene cuando la medida de potencia se realiza en la salida del convertidor. Este efecto probablemente sea debido a la mayor probabilidad de error en la definición de $\delta$ del algoritmo P\&O con medida en la salida del generador por no tener en cuenta las características del convertidor. Aunque debe ser estudiado con más profundidad, es siempre un efecto positivo.

\section{English summary}

\section{CONTROL STRATEGIES}

COMPARISON FOR MPPT SYSTEMS: OPTIMIZATION OF THE DELIVERED TO THE LOAD POWER VS OPTIMIZATION OF THE EXTRACTED FROM THE PHOTOVOLTAIC GENERATOR POWER.

\footnotetext{
Abstract

The maximum power point tracking systems (MPPT) try to maximize the performance of a photovoltaic installation by dynamically adjusting the duty cycle

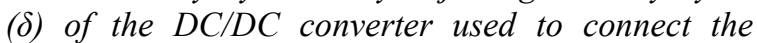
generator to the load. Traditionally, the control algorithm used to define the duty cycle determines the value of this parameter in order to maximize the power generated by the photovoltaic generator. These techniques do not take into account the nonideal character of the DC/DC converter, whose real efficiency also depends on the duty cycle with which it operates. Considering the characteristics of the $D C / D C$ converter can not be said, a priori, that the method that optimizes the power generation of the photovoltaic generator also maximizes the power delivery in the load, the final objective of an MPPT system.
}

This work adapts the well-known algorithm "Perturbation / Observation, $P \& O$ " to optimize the delivery of power in the load, measuring the variables that determine the behavior of the algorithm at the output of the DC / DC converter, and compares its performance with the performance that it is obtained with the classical approach of optimizing the power delivered by the photovoltaic generator, in which the measurement of the variables that determine the operation of the algorithm are made at the input of the DC / DC converter.

A photovoltaic system that uses a boost converter to connect the photovoltaic generator with the load has been analyzed, which has been subjected to variable atmospheric conditions, in terms of radiation and temperature, and to different load consumption requirements, obtaining the performances offered by the system for the two control philosophies. These results are discussed to determine if there is a significant loss of performance in the classical approach with respect to the new proposal and the convenience of developing systems that optimize the useful power, that is, the power delivered to the load.

Keywords: MPPT, converter, duty cycle, Photovoltaic generator, efficience.

\section{Referencias}

[1] Enrique J. M., Durán E., Sidrach M., Andújar J. (2007). M. Theoretical Assessment of the Maximum Power Point Tracking Efficiency of Photovoltaic Facilities With Different Converter Topologies. Solar Energy, 81(1), pp. 31-38.

[2] Enrique J. M.; Andújar J. M.; Durán E.; Martínez M. A. (2015). Maximum-Power Point Tracker based on Maximum-Power Point Resistance Modeling. Progress in Photovoltaics. Vol. 23, pp. 1.940-1.955.

[3] Enrique J.M., Andújar J.M., Martínez M.A. (2010). Reliable, Fast and Low Cost Maximum Power Point Tracker for Photovoltaic Applications. Solar Energy. Vol. 84. Núm. 1. Pag. 79-89.

[4] Hohm D. P., Ropp M. E. (2002).Comparative Study of Maximum Power Point Tracking Algorithms. Progress in Photovoltaics: Research and applications.

[5] Abd El-Shafy A.N., Faten H.F., Abou El-Zahab E.M. (2003). Evaluation of a proper controller performance for maximum-power point tracking of a stand-alone PV system. Solar Energy Mater. Solar Cells 75 (3-4), pp. 723728. 
[6] Hiyama T., et al. (1995). Evaluation of neural network based real time maximum power tracking controller for PV system. IEEE Trans. Energy Conversion, 10 (3), pp. 543-548.

[7] Hiyama T., et al. (1995). Identification of optimal operation point of PV modules using neural network for real time maximum power tracking control. IEEE Trans. Energy Conversion 10, pp 360-367.

[8] Patcharaprakiti N., Premrudeepreechacharn S., Sriuthaisiriwong Y. (2005). Maximum power point tracking using adaptive fuzzy logic control for grid-connected photovoltaic system. Renewable Energy, 30 (11), pp. 1771-1788.

[9] Takashima T., Tanaka T., Amano M., Ando Y. (2000). Maximum output control of photovoltaic (PV) array. Intersociety Energy Conversion Engineering Conference and Exhibit (IECEC), 35th, Las Vegas, NV, 24-28, pp. 380-383.

[10] Veerachary M., Senjyu T., Uezato K. (2003). Maximum power point tracking of coupled inductor interleaved boost converter supplied PV system. IEE Proc.-Electr. Power Appl., Vol. 150. No. 1.

[11] Enrique J.M. , Barragán A.J., Duran E., Andújar J.M. (2018). Theoretical Assessment of DC/DC Power Converters' Basic Topologies. A Common Static Model. Appl. Sci. 2018.

[12] Enrique J.M. et al. . (2018). Comparative Analysis of the Tracking Efficiency of a Maximum-Power Point Tracker Based on Maximum Power Point Resistance Modeling Versus a Classic P\&O. In: Mortal A. et al. (eds) INCREaSE. INCREaSE 2017, Faro (Portugal).Springer, Cham

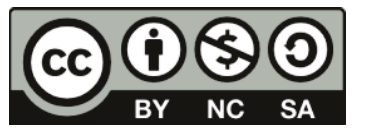

(C) 2019 by the authors. Submitted for possible open access publication under the terms and conditions of the Creative Commons Attribution CC BY-NC-SA 4.0 license (https://creativecommons.org/licenses/bync-sa/4.0/deed.es). 